TRANSACTIONS OF THE

AMERICAN MATHEMATICAL SOCIETY

Volume 354, Number 7 , Pages 2955-2969

S 0002-9947(02)02988-4

Article electronically published on February 25, 2002

\title{
COMMENSURABILITY OF 1-CUSPED HYPERBOLIC 3-MANIFOLDS
}

\author{
DANNY CALEGARI AND NATHAN M. DUNFIELD
}

\begin{abstract}
We give examples of non-fibered hyperbolic knot complements in homology spheres that are not commensurable to fibered knot complements in homology spheres. In fact, we give many examples of knot complements in homology spheres where every commensurable knot complement in a homology sphere has non-monic Alexander polynomial.
\end{abstract}

\section{INTRODUCTION}

For over 20 years, progress in 3-manifold theory has been stimulated by:

1.1. Thurston's Virtual Fibration Conjecture. Let $M$ be a finite volume hyperbolic 3-manifold. Then $M$ has a finite cover which is a surface bundle over $S^{1}$.

Little progress has been made towards the resolution of this conjecture since it was proposed Thu. In fact, there are few 3-manifolds which do not fiber over $S^{1}$ but are known to have finite covers which do fiber (see the references in Kir Prob. 3.51]). Moreover, Boileau and Wang [BW] produced infinitely many examples of closed hyperbolic 3-manifolds for which no solvable cover fibers over $S^{1}$. However, fundamental groups of hyperbolic 3-manifolds have huge numbers of finite index subgroups with a variety of quotients [Lub, and many 3-manifolds fiber over $S^{1}$ in many different ways. Thus it is likely that more complicated classes of covers provide numerous examples.

A relatively tractable class of 3-manifolds are knot complements in $S^{3}$, or more generally, knot complements in rational homology spheres. If such a 3 -manifold fibers over $S^{1}$, the fiber is a minimal genus Seifert surface of the knot. Complements of knots in rational homology spheres rarely cover each other, but much more frequently they share a common finite cover - that is, they are commensurable. For this class of manifolds, the natural analogue of Thurston's question is:

1.2. Question. Let $M$ be a knot complement in a rational homology sphere. When is $M$ commensurable with a fibered knot complement in a rational homology sphere?

Here, we give conditions on a knot complement which ensure that it is not commensurable with a fibered knot complement. These conditions are satisfied in

Received by the editors February 7, 2001 and, in revised form, August 25, 2001.

2000 Mathematics Subject Classification. Primary 57M25, 57M50.

Key words and phrases. Virtual Fibration Conjecture, commensurability, Alexander polynomial, character variety.

Both authors were partially supported by the National Science Foundation.

(C)2002 American Mathematical Society 
many examples, including the complements of a large number of 2-bridge knots in $S^{3}$. These conditions are somewhat subtle, but we give examples showing that the subtleties are essential.

Recall the basic:

1.3. Criterion. Let $M$ be a knot complement in a rational homology sphere. If the Alexander polynomial $\Delta_{M}$ of $M$ is not monic, then $M$ does not fiber over $S^{1}$.

Our main idea is to combine this criterion with the fact that the roots of $\Delta_{M}$ are related to eigenvalues of reducible $\mathrm{PSL}_{2} \mathbb{C}$-representations of $\pi_{1}(M)$.

We'll now state the main result. A 1-cusped hyperbolic 3-manifold $M$ is generic if it is not arithmetic and its commensurator orbifold has a flexible cusp. The latter condition holds if the cusp shape of $M$ is not in $\mathbb{Q}(i)$ or $\mathbb{Q}(\sqrt{-3})$. Our condition concerns reducible representations $\rho: \pi_{1}(M) \rightarrow \mathrm{PSL}_{2} \mathbb{C}$ whose image is non-abelian. A representation $\rho: \pi_{1}(M) \rightarrow \mathrm{PSL}_{2} \mathbb{C}$ is called integral if the trace of $\rho(\gamma)$ is an algebraic integer for all $\gamma \in \pi_{1}(M)$. Otherwise, $\rho$ is non-integral. As discussed in Section 5, whether $M$ has a non-abelian reducible representation which is non-integral is closely related to whether $\Delta_{M}$ has a non-integral root. When $M$ fibers, its Alexander polynomial $\Delta_{M}$ is monic, that is, has lead coefficient \pm 1 , and so all the roots are integral. Using the connection between $\Delta_{M}$ and reducible representations, it is easy to show that every non-abelian reducible representation of $\pi_{1}(M)$ is integral.

Let $X_{0}(M)$ denote the geometric component of the $\mathrm{PSL}_{2} \mathbb{C}$-character variety of $\pi_{1}(M)$ (see Section 3. We will show:

6.1. Theorem. Let $M_{1}$ be a generic hyperbolic knot complement in a $\mathbb{Z} / 2 \mathbb{Z}$ homology sphere. Suppose that the geometric component $X_{0}\left(M_{1}\right)$ contains the character of a non-integral reducible representation. Then $M_{1}$ is not commensurable to a fibered knot complement in a $\mathbb{Z} / 2 \mathbb{Z}$-homology sphere.

Suppose $M_{1}$ and $M_{2}$ are commensurable manifolds. Given a representation $\rho_{1}: \pi_{1}\left(M_{1}\right) \rightarrow \mathrm{PSL}_{2} \mathbb{C}$ one cannot in general induce a representation $\rho_{2}: \pi_{1}\left(M_{2}\right) \rightarrow$ $\mathrm{PSL}_{2} \mathbb{C}$ which is compatible, that is, agrees with $\rho_{1}$ on the fundamental group of the common cover of $M_{1}$ and $M_{2}$. However, when $M_{1}$ and $M_{2}$ are generic commensurable 1-cusped hyperbolic 3-manifolds one knows more. Here, the existence of a commensurator [Bor], together with Thurston's Hyperbolic Dehn Surgery Theorem Thu], gives a natural birational isomorphism between the geometric components of the character varieties of $M_{1}$ and $M_{2}$ (this is due to the first author (unpublished) and [LR ). Moreover, representations of $\pi_{1}\left(M_{1}\right)$ coming from its geometric component $X_{0}\left(M_{1}\right)$ correspond to compatible representations of $\pi_{1}\left(M_{2}\right)$ coming from its geometric component $X_{0}\left(M_{2}\right)$. The key to Theorem 6.1 is showing that for a reducible representation of $\pi_{1}\left(M_{1}\right)$ in $X_{0}\left(M_{1}\right)$, the corresponding compatible representation of $\pi_{1}\left(M_{2}\right)$ is also reducible. Then if $X_{0}\left(M_{1}\right)$ contains the character of a non-integral reducible representation, there is a corresponding reducible representation of $\pi_{1}\left(M_{2}\right)$. This representation has to be non-integral as well, and so $M_{2}$ can't be fibered.

We end this section with an outline of the rest of the paper. In Section 2] we give basic topological restrictions on when fibered and non-fibered 1-cusped manifolds can be commensurable. We also provide constructions of pairs of commensurable 
1-cusped manifolds satisfying these restrictions. Section 3 3 contains background material about character varieties. Section 4 discusses representations of commensurable 3-manifolds. Section 5 discusses the Alexander polynomial and its connection to reducible representations. Section [6] is devoted to the proof of Theorem 6.1.

In Section 7 we show that Theorem 6.1 applies to the complements of many 2-bridge knots, in particular, to all non-fibered 2-bridge knots $K(p, q)$ where $q<$ $p<40$.

In Section 8, we give examples of pairs of commensurable 1-cusped hyperbolic 3 -manifolds exactly one of which fibers. These illustrate the necessity and subtlety of the hypotheses of Theorem 6.1.

Acknowledgements. The first author was partially supported by an NSF Graduate Fellowship and an NSF VIGRE grant. The second author was partially supported by an NSF Postdoctoral Fellowship. We also thank the referee for helpful comments, especially those clarifying the definition of an integral representation.

\section{Commensurability of 1-CuSPed 3-Manifolds}

In this section, we'll discuss some basic necessary conditions for a 1-cusped nonfibered 3-manifold to be commensurable with a 1-cusped fibered 3-manifold. We'll also describe some constructions of pairs of commensurable manifolds which show that these necessary conditions are satisfied in many examples.

Let $M$ be a 3 -manifold. Given $A$ in $H^{1}(M ; \mathbb{Z})$ we can think of it in several ways: as a homomorphism of $\pi_{1}(M)$ to $\mathbb{Z}$, as a homotopy class of maps from $M$ to $S^{1}$, or as a surface representing a class in $H_{2}(M, \partial M)$ (via Lefschetz duality). Thought of as a class of maps from $M$ to $S^{1}$, it makes sense to say that $A$ is representable by a fibration over $S^{1}$.

We'll begin with the question: Suppose $M$ is a 3-manifold which does not fiber over $S^{1}$; when does a finite cover of $M$ fiber? The fundamental fact here is:

2.1. Lemma (Stallings). Suppose $M$ is an orientable 3-manifold which does not fiber over $S^{1}$. Let $N$ be a finite cover which does fiber over $S^{1}$, with $\phi: N \rightarrow M$ the covering map. If $A$ is a class in $H^{1}(M ; \mathbb{Z})$, then the pullback $\phi^{*}(A)$ in $H^{1}(N ; \mathbb{Z})$ cannot represent a fibration over $S^{1}$. In particular,

$$
\operatorname{rank} H^{1}(N)>\operatorname{rank} H^{1}(M) .
$$

Proof. Let $A$ be in $H^{1}(M, \mathbb{Z})$. Stallings showed that $A$ can be represented by a fibration over $S^{1}$ if and only if the kernel of the map $A: \pi_{1}(M) \rightarrow \mathbb{Z}$ is finitely generated [Sta]. So as $M$ does not fiber, the kernel of the $A: \pi_{1}(M) \rightarrow \mathbb{Z}$ is not finitely generated. As $\pi_{1}(N)$ has finite index in $\pi_{1}(M)$, it follows that the kernel of the restricted map $\phi^{*}(A): \pi_{1}(N) \rightarrow \mathbb{Z}$ is also not finitely generated. So $\phi^{*}(A)$ cannot represent a fibration.

Now suppose two manifolds $M_{1}$ and $M_{2}$ are commensurable, that is, they have a common finite sheeted cover $N$. The following theorem gives a restriction on when a non-fibered 1-cusped 3-manifold can be commensurable to a fibered one:

2.2. Theorem. Let $M_{1}$ and $M_{2}$ be two commensurable hyperbolic knot complements in rational homology spheres. Suppose $M_{2}$ fibers over $S^{1}$ but $M_{1}$ does not. Then a common regular cover must have at least 2 boundary components. 
Proof. First, let's make some basic observations. Throughout, all (co)homology will have coefficients in $\mathbb{Z}$. Suppose $\phi: N \rightarrow M$ is a regular finite cover of 3-manifolds. Let $G=\pi_{1}(M) / \pi_{1}(N)$ be the covering group. The homomorphism $\phi^{*}: H^{1}(M) \rightarrow$ $H^{1}(N)$ is injective, and $\phi^{*}\left(H^{1}(M)\right)$ is exactly the $G$-invariant cohomology.

Now let's prove the theorem. Suppose $N$ is a common regular cover of $M_{1}$ and $M_{2}$. Let $\phi_{i}: N \rightarrow M_{i}$ be the covering maps, and $G_{i}=\pi_{1}\left(M_{i}\right) / \pi_{1}(N)$ the covering groups. By Mostow rigidity, we can assume that the covering groups $G_{i}$ act via isometries of some fixed hyperbolic metric on $N$. As the isometry group of $N$ is finite, so is the group $G=\left\langle G_{1}, G_{2}\right\rangle$.

From now on, assume that $N$ has only one cusp. We will show there is a non-zero $G$-invariant class $A$ in $H^{1}(N)$. This gives a contradiction for the following reason. Every non-zero class in $\phi_{2}^{*}\left(H^{1}\left(M_{2}\right)\right)$ can be represented by a fibration, while by Lemma 2.1 no non-zero class in $\phi_{1}^{*}\left(H^{1}\left(M_{1}\right)\right)$ can be represented by a fibration. But because $A$ is $G$-invariant, $A$ is in $\phi_{i}^{*}\left(H^{1}\left(M_{i}\right)\right)$ for both $i$, which is impossible.

Let $S$ be a Seifert surface for $M_{2}$ which is a fiber, and let $F=\phi_{2}^{-1}(S)$ be the lift to $N$. The surface $F$ represents a non-trivial class in $H_{2}(N, \partial N)$. Moreover, since $[\partial S]$ in $H_{1}\left(\partial M_{2}\right)$ is nontrivial, so is $[\partial F]$ in $H_{1}(\partial N)$. Look at the the class in $H_{2}(N, \partial N)$ which is

$$
X=\sum_{g \in G} g_{*}([F]) .
$$

Consider the restricted coverings $\phi_{i}: \partial N \rightarrow \partial M_{i}$. Now the covering group $G_{i}$ acts freely on the torus $\partial N$. Hence $\phi_{i}$ induces a rational isomorphism on $H_{1}$, and $G_{i}$ acts identically on $H_{1}(\partial N ; \mathbb{Z})$. Therefore $G$ acts identically on $H_{1}(\partial N ; \mathbb{Z})$. Thus

$$
\partial X=\sum_{g \in G} g_{*}([\partial F])=|G| \cdot[\partial F] \neq 0 .
$$

So $X$ is non-zero. If $A$ is the dual class in $H^{1}(N)$, then $A$ is the non-zero $G$-invariant class we sought.

2.3. Remark. Suppose $M_{1}$ and $M_{2}$ have a common manifold quotient $M$ with a torus cusp. In this case, there are Seifert surfaces generating $H_{2}\left(M_{i}, \partial M_{i}\right)$ which are pullbacks of a single Seifert surface in $M$. In particular, if $M_{2}$ fibers but $M_{1}$ does not, the commensurator cannot be a manifold, but must be an orbifold.

2.4. Example. Suppose $M_{1}$ and $M_{2}$ have a common finite regular cover $N$ with covering deck groups $G_{1}$ and $G_{2}$. If the $M_{i}$ are not hyperbolic, it is not always true that there are actions $G_{i}^{\prime}$ isotopic to $G_{i}$ so that $G=\left\langle G_{1}^{\prime}, G_{2}^{\prime}\right\rangle$ is finite. For example, let $M_{1}$ be the unit tangent bundle of the $(2,4,4)$ Euclidean triangle orbifold, and $M_{2}$ the unit tangent bundle of the $(2,3,6)$ Euclidean triangle orbifold. These manifolds have $T^{3}$ as a common regular cover, with deck groups $G_{1} \cong \mathbb{Z} / 4 \mathbb{Z}$ and $G_{2} \cong \mathbb{Z} / 6 \mathbb{Z}$ respectively. But the action of $\left\langle G_{1}, G_{2}\right\rangle$ on $H_{1}\left(T^{3} ; \mathbb{Z}\right)$ generates a group isomorphic to $S L(2, \mathbb{Z})$. Thus, we can't isotope the $G_{i}$ so that together they generate a finite group.

In light of Theorem 2.2 it is worth producing examples of commensurable 1cusped manifolds whose common covers have multiple cusps.

2.5. Example. Let $N_{0}=T^{2} \times I$. There are two orientation-preserving involutions $\phi_{1}, \phi_{2}$ of $N_{0}$ defined by

$$
\phi_{1}(x, y, t)=(-x, y, 1-t), \quad \phi_{2}(x, y, t)=(x,-y, 1-t)
$$


where $(x, y)$ are angular coordinates on $T^{2}$ and $t$ is the coordinate on $I=[0,1]$.

Now, the union of the fixed point sets of $\phi_{1}$ and $\phi_{2}$ is a graph $\Gamma \subset T^{2} \times 1 / 2$. A regular neighborhood $N(\Gamma)$ of $\Gamma$ is a genus 5 handlebody. Let $N_{1}=N_{0}-N(\Gamma)$, and let $N_{2}$ be the double of $N_{1}$. Then $N_{2}$ has two sets of cusps, $\left\{C_{1}, C_{2}\right\}$ and $\left\{D_{1}, D_{2}\right\}$, where $C_{1}$ and $C_{2}$ are the original pair of cusps from $N_{0}$. The involutions $\phi_{1}$ and $\phi_{2}$ extend to fixed-point-free involutions of $N_{2}$ which interchange $C_{1}$ with $C_{2}$ and $D_{1}$ with $D_{2}$. Notice that

$$
\phi_{2} \phi_{1}^{-1}: T^{2} \times 0 \rightarrow T^{2} \times 0
$$

is just the involution $(x, y) \rightarrow(-x,-y)$. It follows that these involutions descend to the manifold obtained by equivariantly Dehn filling $D_{1}$ and $D_{2}$. Let $N_{3}$ be obtained from $N_{2}$ by Dehn filling on both $D_{1}$ and $D_{2}$, so that $\phi_{1}$ and $\phi_{2}$ both act on $N_{3}$. We can also equivariantly surger $N_{3}$ along some collection of links to destroy any "accidental" additional symmetry to get $N_{3}^{\prime}$, so that the quotients $M_{1}=N_{3}^{\prime} / \phi_{1}$ and $M_{2}=N_{3}^{\prime} / \phi_{2}$ are not homeomorphic, and have no common regular cover with fewer than two cusps. This equivariant surgery can also be used to kill off rational homology and ensure $N_{3}$ is hyperbolic, so that $M_{1}$ and $M_{2}$ can be chosen to be knot complements in rational homology spheres.

These examples have the property that for $S$ a Seifert surface in $M_{2}$, the class [ $\left.\phi_{1} \phi_{2}^{-1}(S)\right]$ is trivial in $H_{2}\left(M_{1}, \partial M_{1}\right)$. So the proof of Theorem [2.2 does not apply here, and we cannot conclude anything about whether $M_{1}$ and $M_{2}$ mutually fiber or do not fiber over $S^{1}$.

These examples cannot be chosen to be knot complements in $\mathbb{Z} / 2 \mathbb{Z}$-homology spheres because of the very existence of a 2 -fold cover. A modification of this construction gets around this difficulty.

2.6. Example. Let $L$ be the unlink in $S^{3}$ on $n$ components. Arrange these symmetrically so that there is a rotation $r$ with axis $\alpha$ permuting the components of $L$. Let $s$ be a rotation of order $n$ fixing each component, translating $\alpha$, and fixing another axis $\beta$ which links each component of $L$. Let $\phi_{1}=r s$ and $\phi_{2}=r s^{k}$ for some $k>1$ coprime with $n$. Then $M_{i}=\left(S^{3}-L\right) / \phi_{i}$ is a knot complement in a lens space which is a $\mathbb{Z} / 2 \mathbb{Z}$-homology sphere for $n$ odd. By equivariant surgery, we can make the $M_{i}$ hyperbolic knot complements in $\mathbb{Z} / 2 \mathbb{Z}$-homology spheres whose smallest common cover has $n$ cusps.

\section{Character varieties}

Here, we review the part of the theory of character varieties of 3-manifolds that we will need for Theorem 6.1. For details, see [CS, Sha. For the technicalities of the $\mathrm{PSL}_{2} \mathbb{C}$ as opposed to $\mathrm{SL}_{2} \mathbb{C}$ case see [BZa].

3.1. Definition. For $M$ a compact 3-manifold, we let

$$
R(M)=\operatorname{Hom}\left(\pi_{1}(M), \mathrm{PSL}_{2} \mathbb{C}\right)
$$

denote the $\mathrm{PSL}_{2} \mathbb{C}$-representation variety of $M$.

Further, let

$$
X(M)=\bigcup_{\rho \in R(M)} \operatorname{tr}_{\rho}^{2} \subseteq \mathbb{C}^{\pi_{1}(M)}
$$

denote the $\mathrm{PSL}_{2} \mathbb{C}$-character variety of $M$. 
Since $\pi_{1}(M)$ is finitely generated, $R(M)$ is an affine algebraic variety over $\mathbb{C}$. For each $\gamma \in \pi_{1}(M)$, the function $\tau_{\gamma}: R(M) \rightarrow \mathbb{C}$ defined by

$$
\tau_{\gamma}(\rho)=\operatorname{tr}_{\rho}^{2}(\gamma)
$$

is a regular function on $R(M)$. As $\pi_{1}(M)$ is finitely generated, the ring of functions generated by the $\tau_{\gamma}$ is finitely generated. It follows that $X(M)$ in $\mathbb{C}^{\pi_{1}(M)}$ projects isomorphically to an algebraic subvariety of $\mathbb{C}^{G}$ for some finite subset $G \subset \pi_{1}(M)$. Therefore $X(M)$ has the structure of an affine algebraic variety over $\mathbb{C}$.

Away from the reducible locus, the action of $\mathrm{PSL}_{2} \mathbb{C}$ on $R(M)$ by conjugation is transverse, and the natural projection $R(M) / \mathrm{PSL}_{2} \mathbb{C} \rightarrow X(M)$ is injective on a Zariski open set. Let $t: R(M) \rightarrow X(M)$ be the projection. For a character $\chi$ in $X(M), t^{-1}(\chi)$ either consists solely of the conjugates of a single irreducible representation, or $t^{-1}(\chi)$ consists of reducible representations. In the latter case, the reducible representations in $t^{-1}(\chi)$ may not all be conjugate (it is easy to see that the closure of the orbit of a non-abelian reducible representation contains an abelian reducible representation).

We will need the following, which is Proposition 6.2 of CGLS].

3.2. Lemma. Let $X$ be an irreducible component of $X(M)$ which contains the character of an irreducible representation. Let $\chi \in X$ be the character of a reducible representation. Then there is a non-abelian reducible representation with character $\chi$.

The lemma follows from the fact that the fibers of $t$ over $X$ are all at least 3 -dimensional whereas the orbit under conjugation of an abelian reducible representation is 2-dimensional.

If $M$ is an orientable finite volume hyperbolic 3-manifold, there is a unique discrete faithful representation

$$
\rho_{\delta}: \pi_{1}(M) \rightarrow \mathrm{PSL}_{2} \mathbb{C}
$$

up to conjugacy in $O(3,1) \cong \mathrm{PSL}_{2} \mathbb{C} \rtimes \mathbb{Z} / 2 \mathbb{Z}$. Up to conjugacy in $\mathrm{PSL}_{2} \mathbb{C}$, there are a pair of such representations which differ by complex conjugation, and their characters may occur in different irreducible components of $X(M)$. Fixing an orientation of $M$ fixes a $\mathrm{PSL}_{2} \mathbb{C}$-conjugacy class of discrete faithful representations. We will assume our manifolds are oriented in what follows, and therefore that $\rho_{\delta}$ is well-defined up to conjugacy in $\mathrm{PSL}_{2} \mathbb{C}$.

3.3. Definition. Let $M$ be a finite volume hyperbolic 3-manifold. Let $X_{0}(M)$ denote the irreducible component of $X(M)$ containing the character of the discrete faithful representation $\rho_{\delta}$. The component $X_{0}(M)$ is called the geometric component of $X(M)$.

\section{REPRESENTATIONS OF COMMENSURABLE MANIFOLDS}

Let $M_{1}$ and $M_{2}$ be commensurable hyperbolic 3-manifolds with common finite cover $N$. Two representations $\rho_{i}: \pi_{1}\left(M_{i}\right) \rightarrow \mathrm{PSL}_{2} \mathbb{C}$ are said to be compatible if they agree on $\pi_{1}(N)$. For instance, if $\rho_{1}$ is a discrete faithful representation for $M_{1}$, then Mostow rigidity implies that there is a discrete faithful representation $\rho_{2}$ of $\pi_{1}\left(M_{2}\right)$ which is compatible with $\rho_{1}$. The property of having compatible representations extends to other representations whose characters are in $X_{0}\left(M_{1}\right)$. 
4.1. Proposition. Suppose $M_{1}$ and $M_{2}$ are generic commensurable orientable hyperbolic 3-manifolds with one cusp. Let $\chi_{1}$ be a character in the geometric component $X_{0}\left(M_{1}\right)$. Then there exist compatible representations $\rho_{i}$ of $\pi_{1}\left(M_{i}\right)$ such that $\rho_{1}$ has character $\chi_{1}$ and the character of $\chi_{2}$ lies in a geometric component $X_{0}\left(M_{2}\right)$.

The reason for weaseling around with $\chi_{1}$ instead of just starting with $\rho_{1}$ is that for characters of reducible representations, there can be different conjugacy classes of representations with that character. It turns out that you can't always specify $\rho_{1}$, but only $\chi_{1}$, in this case.

Proof. As the $M_{i}$ are non-arithmetic, they cover a common orientable commensurator orbifold $Q$ [Bor]. Let $p_{i}: M_{i} \rightarrow Q$ be the (orbifold) covering maps. The inclusion of $\pi_{1}\left(M_{i}\right)$ into $\pi_{1}(Q)$ induces a map $p_{i_{*}}: X_{0}(Q) \rightarrow X_{0}\left(M_{i}\right)$ via restriction of representations. Because the $M_{i}$ are generic, $Q$ has a flexible cusp, and the variety $X_{0}(Q)$ is also a complex curve. In fact, $p_{i_{*}}$ is a birational isomorphism, though we will not need this [LR]. The main step is:

4.2. Lemma. The map $p_{1 *}: X_{0}(Q) \rightarrow X_{0}\left(M_{1}\right)$ is onto.

Proof of Lemma. The map $p_{1 *}$ is a non-constant map of irreducible affine algebraic curves over $\mathbb{C}$. Let $\bar{X}_{0}(Q)$ denote the smooth projective model of $X_{0}(Q)$. The curve $\bar{X}_{0}(Q)$ is the normalization of $X_{0}(Q)$ compactified by adding an ideal point for each end of $X_{0}(Q)$ CGLS, §1.5]. Similarly, let $\bar{X}_{0}\left(M_{1}\right)$ be the smooth projective model of $X_{0}\left(M_{1}\right)$. The map $p_{1 *}$ induces a regular map of the same name between $\bar{X}_{0}(Q)$ and $\bar{X}_{0}\left(M_{1}\right)$ (this map is just a branched covering of closed Riemann surfaces). Let $\chi_{1}$ be a point in $\bar{X}_{0}\left(M_{1}\right)$ which corresponds to a character - that is, not an ideal point. As the map from $\bar{X}_{0}(Q)$ to $\bar{X}_{0}\left(M_{1}\right)$ is surjective, choose $\chi_{0}$ in $\bar{X}_{0}(Q)$ with $p_{1 *}\left(\chi_{0}\right)=\chi_{1}$. We need to show that $\chi_{0}$ is not an ideal point. Suppose that $\chi_{0}$ is an ideal point. By Proposition 1.4.4 of CS there is some $\gamma$ in $\pi_{1}(Q)$ for which $\operatorname{tr}_{\gamma}^{2}\left(\chi_{0}\right)=\infty$. That is, there is some element of $\pi_{1}(Q)$ which acts by a hyperbolic isometry on the simplicial tree associated to the ideal point $\chi_{0}$. Now for any $n>0$,

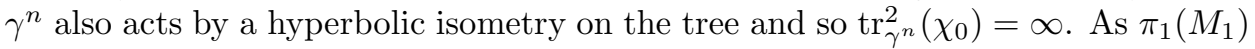
is of finite index in $\pi_{1}(Q)$, we can choose $n$ so that $\gamma^{n}$ is in $\pi_{1}\left(M_{1}\right)$. But then $\operatorname{tr}_{\gamma^{n}}^{2} \chi_{0}=\operatorname{tr}_{\gamma^{n}}^{2} \chi_{1}=\infty$, contradicting that $\chi_{1}$ is the character of a representation. So $\chi_{0}$ is not an ideal point and hence $p_{1_{*}}: X_{0}(Q) \rightarrow X_{0}\left(M_{1}\right)$ is onto.

Now to finish the proof of the theorem, let $\chi_{1} \in X_{0}\left(M_{1}\right)$. By the lemma, there is some character $\chi_{0}$ in $X_{0}(Q)$ with $p_{1 *}\left(\chi_{0}\right)=\chi_{1}$. Let $\rho_{0}$ be a representation with character $\chi_{0}$. Then the restrictions of $\rho_{0}$ to the subgroups $\pi_{1}\left(M_{i}\right)$ give a pair of compatible representations with the required properties.

\section{The Alexander polynomial and Reducible Representations}

Let $M$ be a knot complement in a rational homology sphere. Let $N$ denote the universal free abelian cover of $M$. That is, set $H=H_{1}(M ; \mathbb{Z}) /($ torsion) and take the covering corresponding to the kernel of the natural homomorphism $\pi_{1}(M) \rightarrow H$. Then $N$ is a regular covering of $M$, and the group $\pi_{1}(M) / \pi_{1}(N)=H \cong \mathbb{Z}$ acts on $N$ by deck transformations. It follows that $H_{1}(N ; \mathbb{Z})$ has the natural structure of a $\mathbb{Z}[H]$-module. If $t$ denotes the generator of $H$, then $H_{1}(N ; \mathbb{Z})$ is a $\mathbb{Z}\left[t, t^{-1}\right]$-module. The Alexander polynomial $\Delta_{M}$ of $M$ is an invariant of this module. In the case that $H_{1}(N ; \mathbb{Z})$ is cyclic, that is $H_{1}(N ; \mathbb{Z})=\mathbb{Z}\left[t, t^{-1}\right] / p(t)$, the polynomial $\Delta_{M}$ is just $p(t)$. In general, $\Delta_{M}$ is the greatest common divisor of the 0 -th elementary ideal of a matrix which presents $H_{1}(N ; \mathbb{Z})$ as a $\mathbb{Z}\left[t, t^{-1}\right]$-module. The Laurent polynomial 
$\Delta_{M}$ is only defined up to multiplication by a unit $\pm t^{n}$ in $\mathbb{Z}\left[t, t^{-1}\right]$. A key property for us is that the Alexander polynomial is symmetric, that is $\Delta_{M}\left(t^{-1}\right)= \pm t^{n} \Delta_{M}(t)$ Tur. For more on the Alexander polynomial see [Rol].

If $M$ is a surface bundle over $S^{1}$ with fiber $F$ and monodromy $\phi: F \rightarrow F$, then $N=F \times \mathbb{R}$ and the action of $t$ on $H_{1}(N ; \mathbb{Z})$ is exactly equal to the action of $\phi_{*}$ on $H_{1}(F ; \mathbb{Z})=H_{1}(N ; \mathbb{Z})$. In this case, $\Delta_{M}$ is the characteristic polynomial of $\phi_{*}$. Since $\phi$ is a homeomorphism, $\phi_{*}$ is an automorphism, and $\Delta_{M}$ is monic.

Now consider a non-abelian reducible representation $\rho: \pi_{1}(M) \rightarrow \mathrm{SL}_{2} \mathbb{C}$. Conjugate $\rho$ so that its image is upper-triangular. Given $\gamma \in \pi_{1}(M)$ we will say that the primary eigenvalue of $\rho(\gamma)$ is the $(1,1)$ entry of the matrix $\rho(\gamma)$. This is well-defined for the following reason. Since $\rho$ has non-abelian image, there is a unique line $L$ in $\mathbb{C}^{2}$ which is left invariant by all $\rho\left(\pi_{1}(M)\right)$. The primary eigenvalue of $\rho(\gamma)$ is just the eigenvalue of $\rho(\gamma)$ with eigenspace $L$.

A reducible representation into $\mathrm{SL}_{2} \mathbb{C}$ has meta-abelian (two-step solvable) image. The Alexander polynomial of $M$ is an invariant of the maximal meta-abelian quotient of $\pi_{1}(M)$, so it's not surprising that it is related to non-abelian reducible representations of $\pi_{1}(M)$ into $\mathrm{SL}_{2} \mathbb{C}$. For knots in $\mathbb{Z}$-homology spheres, the statement is:

5.1. Theorem (de Rham). Let $M$ be a knot complement in a $\mathbb{Z}$-homology sphere. Let $\mu$ in $\pi_{1}(\partial M)$ be a meridian. The following are equivalent:

- There is a non-abelian reducible representation

$$
\rho: \pi_{1}(M) \rightarrow \mathrm{SL}_{2} \mathbb{C}
$$

such that $\rho(\mu)$ has primary eigenvalue $m$.

- $m^{2}$ is a root of $\Delta_{M}(t)$.

More generally, for knots in $\mathbb{Q}$-homology spheres there is a similar connection that is a bit harder to state. Let $\rho: \pi_{1}(M) \rightarrow \mathrm{SL}_{2} \mathbb{C}$ be a non-abelian reducible representation. Then $\rho$ acts on $\mathbb{C P}^{1}$ and has a unique fixed point. Translating that point to $\infty, \rho$ can be interpreted as a homomorphism from $\pi_{1}(M)$ into the (complex) affine group of $\mathbb{C}$ :

$$
\text { Affine }(\mathbb{C})=\{\operatorname{maps} z \mapsto a z+b\} \cong\left\{\left(\begin{array}{ll}
a & b \\
0 & 1
\end{array}\right)\right\} \quad \text { where } a \in \mathbb{C}^{\times} \text {and } b \in \mathbb{C} \text {. }
$$

Define a homomorphism $x_{\rho}: \pi_{1}(M) \rightarrow \mathbb{C}^{\times}$by setting $x_{\rho}(\gamma)=a$, where $a$ is the homothety part of $\rho(\gamma)$ thought of as an element of Affine $(\mathbb{C})$. Note that $x_{\rho}(\gamma)$ is just the square of the primary eigenvalue of $\rho(\gamma)$ regarded as being in $\mathrm{SL}_{2} \mathbb{C}$. The map $x_{\rho}$ is often called the character of $\rho$ but to prevent confusion we'll avoid this practice. The case of knots in $\mathbb{Q}$-homology spheres is more complicated than the $\mathbb{Z}$ case because not every homomorphism $x: \pi_{1}(M) \rightarrow \mathbb{C}^{\times}$factors through the free abelianization of $\pi_{1}(M)$. For those homomorphisms that do, Theorem 5.1 in this context is just:

5.2. Theorem. Let $M$ be a knot complement in a $\mathbb{Q}$-homology sphere. Let $H$ be the free abelianization of $\pi_{1}(M)$. Let $x: H \rightarrow \mathbb{C}^{\times}$be a homomorphism. Then the following are equivalent:

- There is a non-abelian reducible representation

$$
\rho: \pi_{1}(M) \rightarrow \mathrm{SL}_{2} \mathbb{C}
$$

with $x_{\rho}=x$. 
- $x^{\prime}\left(\Delta_{M}\right)=0$, where $x^{\prime}$ is the map $x$ induces from $\mathbb{Z}[H]$ to $\mathbb{C}$.

For a proof, see e.g. [McM, §3].

5.3. Non-integral reducible representations. From the introduction, recall that a representation $\rho$ of $\pi_{1}(M)$ into $\mathrm{PSL}_{2} \mathbb{C}$ is integral if the trace of every $\rho(\gamma)$ is an algebraic integer. Otherwise $\rho$ is non-integral. Let's reformulate this a little. Consider a matrix $A \in \mathrm{PSL}_{2} \mathbb{C}$. An eigenvalue $\lambda$ of $A$ (well-defined up to sign) is a root of the monic polynomial $x^{2} \pm \operatorname{tr}(A) x+1$. Therefore if $\operatorname{tr}(A)$ is integral, the eigenvalues of $A$ are also algebraic integers. The converse is clearly true, so $A$ has integral trace if and only if both eigenvalues of $A$ are integral. Now if $\lambda$ is an eigenvalue of $A$, then the other eigenvalue is $1 / \lambda$. Thus we see that $A$ has integral trace if and only if the eigenvalues of $A$ are algebraic units. In the terminology of the last section, a reducible representation $\rho$ is integral if and only if the primary eigenvalues of all the $\rho(\gamma)$ are algebraic units. Since the primary eigenvalue of $\rho\left(\gamma^{-1}\right)$ is the inverse of the primary eigenvalue of $\rho(\gamma)$, we see that a reducible representation is integral if and only if every primary eigenvalue is an algebraic integer. If we think of $\rho$ as a homomorphism to Affine( $(\mathbb{C})$, we see that $\rho$ is integral if and only if the homothety of each $\rho(\gamma)$ is integral.

For knots in $\mathbb{Z}$-homology spheres, the Alexander polynomial determines the existence of non-abelian reducible representations which are non-integral:

5.4. Proposition. Let $M$ be a knot complement in a $\mathbb{Z}$-homology sphere. Then $M$ has a non-abelian reducible representation into $\mathrm{PSL}_{2} \mathbb{C}$ which is non-integral if and only if $\Delta_{M}$ is not monic.

Proof. As $M$ is a knot complement in a $\mathbb{Z} / 2 \mathbb{Z}$-homology sphere, the cohomology group $H^{2}(M ; \mathbb{Z} / 2 \mathbb{Z})$ vanishes and every representation into $\mathrm{PSL}_{2} \mathbb{C}$ lifts to $\mathrm{SL}_{2} \mathbb{C}$, so we're free to think about $\mathrm{SL}_{2} \mathbb{C}$-representations instead. Consider a reducible representation $\rho$. Since $H_{1}(M)=\mathbb{Z}$ is generated by $\mu$, we see that the primary eigenvalue of any $\rho(\gamma)$ is a power of the primary eigenvalue of $\rho(\mu)$. Thus, it follows from Theorem 5.1 that $M$ has a non-integral reducible representation if and only if $\Delta_{M}$ has a root which is not an algebraic unit.

Suppose that $\Delta_{M}$ is not monic. Then $\Delta_{M}$ has a non-integral root provided that $\Delta_{M}$ is not an integer multiple of a monic integer polynomial. As we're in the $\mathbb{Z}$-homology sphere case, $\Delta_{M}(1)= \pm 1$, and this can't happen. So $\Delta_{M}$ has a nonintegral root, and thus a non-abelian reducible representation which is non-integral.

Now suppose that $\Delta_{M}$ is monic. Then all the roots of $\Delta_{M}$ are algebraic integers. Let $\alpha$ be a root of $\Delta_{M}$. Because $\Delta_{M}$ is symmetric, $1 / \alpha$ is also a root of $\Delta_{M}$ and so is integral. Thus all the roots of $\Delta_{M}$ are algebraic units. So all the nonabelian reducible representations of $\pi_{1}(M)$ are integral, completing the proof of the theorem.

In the general $\mathbb{Z} / 2 \mathbb{Z}$-homology sphere case, there isn't an easy statement like this because Theorem 5.2 only applies to representations coming from certain homomorphisms to $\mathbb{C}^{\times}$. It is true that if $M$ has a non-integral representation, then $\Delta_{M}$ is non-monic (to prove this, a nice point of view is the theory of BNS invariants [Dun, BNS, Bro]). However, if $\Delta_{M}$ is non-monic, $M$ need not have a non-integral reducible representation (e.g. the SnapPea census manifold $m 261$ ). Nor is the above proposition true for the $\mathbb{Z} / 2 \mathbb{Z}$-homology sphere case if one replaces the non-monic hypothesis with "has a non-integral root" (to see that the "only if" direction is false, take the complement of a fibered knot in $S^{3}$ connected sum with a lens space). 
Regardless, the following proposition, which in the $\mathbb{Z}$-homology sphere case follows immediately from Proposition 5.4 and Criterion 1.3 is easy to prove in general.

5.5. Lemma. Let $M$ be a knot complement in a $\mathbb{Z} / 2 \mathbb{Z}$-homology sphere. If $M$ fibers over $S^{1}$ then every non-abelian reducible representation of $\pi_{1}(M)$ into $\mathrm{PSL}_{2} \mathbb{C}$ is integral.

Proof. Let $\rho: \pi_{1}(M) \rightarrow$ Affine $(\mathbb{C})$ be a lift of a given non-abelian reducible PSL $_{2} \mathbb{C}$ representation. As $M$ fibers over $S^{1}$, the universal abelian cover of $M$ is of the form $F \times \mathbb{R}$ where $F$ is a compact surface (here $F$ is some finite abelian cover of a fiber in the fibration of $M)$. As $\pi_{1}(F)$ is the commutator subgroup of $\pi_{1}(M)$, the representation $\rho$ takes $\pi_{1}(F)$ to a finitely generated abelian subgroup $G$ consisting of translations. The subgroup $G$ is non-trivial as $\rho$ is non-abelian. For each $\gamma \in \pi_{1}(M)$ we need to show that if $A=\rho(\gamma)=(z \mapsto a z+b)$ then the homothety $a$ is an algebraic integer. The action of $A$ by conjugation on the normal subgroup $G$ takes an element $(z \mapsto z+\tau)$ to $(z \mapsto z+a \tau)$. So $A$ is a group automorphism of the lattice $G \cong \mathbb{Z}^{n}$. Thought of as an element of $\mathrm{SL}_{n} \mathbb{Z}$, the map $A$ satisfies its characteristic polynomial $f(t)$, which is a monic polynomial with integer coefficients. Let $B=(z \mapsto z+\tau)$ be a non-identity element of $G$. If we act on $B$ via $f(A)$ we get that $f(a) \tau=0$. Thus $f(a)=0$ and $a$ is an algebraic integer. So $\rho$ is integral.

\section{INDUCING REDUCIBLE REPRESENTATIONS OF COMMENSURABLE MANIFOLDS}

This section is devoted to proving:

6.1. Theorem. Let $M_{1}$ be a generic hyperbolic knot complement in a $\mathbb{Z} / 2 \mathbb{Z}$-homology sphere. Suppose that the geometric component $X_{0}\left(M_{1}\right)$ contains the character of a non-integral reducible representation. Then $M_{1}$ is not commensurable to a fibered knot complement in a $\mathbb{Z} / 2 \mathbb{Z}$-homology sphere.

Proof. Suppose that $M_{1}$ is commensurable to another knot complement $M_{2}$ in a $\mathbb{Z} / 2 \mathbb{Z}$-homology sphere. Call the common finite cover $N$. We will show that $M_{2}$ has a non-abelian reducible representation which is non-integral, and so cannot fiber.

Let $\chi_{1}$ in $X_{0}\left(M_{1}\right)$ be the character of a non-integral reducible representation. As $M_{1}$ is generic, by Proposition 4.1, there are representations $\rho_{i}: \pi_{1}\left(M_{i}\right) \rightarrow \mathrm{PSL}_{2} \mathbb{C}$ which agree on $\pi_{1}(N)$ where the character of $\rho_{1}$ is equal to $\chi_{1}$. In particular, $\rho_{1}$ is reducible and non-integral ( $\rho_{1}$ may be abelian, because we don't get to pick $\rho_{1}$, just $\left.\chi_{1}\right)$. Also, the character of $\rho_{2}$ is in $X_{0}\left(M_{2}\right)$.

We will show

6.2. Claim. The representation $\rho_{2}$ of $\pi_{1}\left(M_{2}\right)$ is reducible.

Assuming the claim, let's prove that $M_{2}$ is not fibered. Pick $\gamma$ in $\pi_{1}\left(M_{1}\right)$ such that $\rho_{1}(\gamma)$ has non-integral trace. Then for any $n>0$, the matrix $\rho_{1}\left(\gamma^{n}\right)$ also has non-integral trace as its eigenvalues are powers of those of $\rho_{1}(\gamma)$. Since $\pi_{1}(N)$ is of finite index in $\pi_{1}\left(M_{1}\right)$, choose an $n$ such that $\gamma^{n}$ is in $\pi_{1}(N)$. But then $\gamma^{n}$ is in $\pi_{1}\left(M_{2}\right)$ as well, and so $\rho_{2}\left(\gamma^{n}\right)=\rho_{1}\left(\gamma^{n}\right)$ has non-integral trace. Thus $\rho_{2}$ is non-integral. Since the character of $\rho_{2}$ is in $X_{0}\left(M_{2}\right)$, by Lemma 3.2 there is a non-abelian reducible representation $\rho_{2}^{\prime}$ which has the same character as $\rho_{2}$. As $\rho_{2}^{\prime}$ has the same character as $\rho_{2}$, it is non-integral. By Lemma [5.5 $M_{2}$ does not fiber over $S^{1}$. This completes the proof of the theorem modulo the claim.

Now let's go back and prove Claim 6.2. Let $\Gamma=\pi_{1}\left(M_{2}\right)$ and $\Gamma^{\prime}=\pi_{1}(N)$. Now $\rho_{2}$ restricted to $\Gamma^{\prime}$ is the same as $\rho_{1}$ restricted to $\Gamma^{\prime}$, and $\rho_{1}$ is reducible. Thus $\rho_{2}$ 
is reducible on $\Gamma^{\prime}$. The subgroup $\Gamma^{\prime}$ is of finite index in $\Gamma$, so we can replace it by a finite index normal subgroup of $\Gamma$. Let $G=\rho_{2}(\Gamma)$ and $G^{\prime}=\rho_{2}\left(\Gamma^{\prime}\right)$, two subgroups of $\mathrm{PSL}_{2} \mathbb{C}$. Note that $\Gamma^{\prime}$ is not the trivial subgroup because $\rho_{1}$ is non-trivial, in fact non-integral, on any finite index subgroup of $\pi_{1}\left(M_{1}\right)$.

Now suppose that $\rho_{2}$ is irreducible, that is, the fixed point set of $G$ acting on $\partial \mathbb{H}^{3}$ is empty. As $G^{\prime}$ is reducible, $\operatorname{fix}\left(G^{\prime}\right)$ is either 1 or 2 points. As $G^{\prime}$ is normal in $G$, the set $\operatorname{fix}\left(G^{\prime}\right)$ is $G$-invariant. So if $\operatorname{fix}\left(G^{\prime}\right)$ consisted of a single point, $G$ would be reducible as well. So fix $\left(G^{\prime}\right)$ is 2 points. Look at the homomorphism $h: G \rightarrow \mathbb{Z} / 2 \mathbb{Z}$ where $\mathbb{Z} / 2 \mathbb{Z}$ is thought of as the symmetric group on fix $\left(G^{\prime}\right)$. The homomorphism $h$ is non-trivial as $G$ is irreducible. Any $A \in G$ leaves invariant the geodesic $g$ joining the two points fix $\left(G^{\prime}\right)$. If $h(A)=1$ then $A$ acts on $g$ by an orientation reversing isometry, and $A$ has order 2. Note that $G$ is meta-abelian, as the kernel of $h$ is abelian because it consists of isometries which fix the pair of points fix $\left(G^{\prime}\right)$.

To finish the proof of the claim, we look at $H=\rho_{2}\left(\pi_{1}\left(\partial M_{2}\right)\right)$. We claim that $H$ is finite. Let $\mu_{2}$ in $\pi_{1}\left(\partial M_{2}\right)$ be a meridian, that is, Dehn filling in along $\mu_{2}$ yields a $\mathbb{Z} / 2 \mathbb{Z}$-homology sphere. Let $\lambda_{2}$ in $\pi_{1}\left(\partial M_{2}\right)$ be a longitude, that is, a generator of the kernel $H_{1}\left(\partial M_{2}, \mathbb{Z}\right) \rightarrow H_{1}\left(M_{2}, \mathbb{Z}\right)$. If $M$ were the complement of a knot in a $\mathbb{Z}$-homology sphere, $\left(\mu_{2}, \lambda_{2}\right)$ would be a basis of $\pi_{1}\left(\partial M_{2}\right)$. In general, $\left(\mu_{2}, \lambda_{2}\right)$ generate a finite index subgroup of $\pi_{1}\left(\partial M_{2}\right)$. As $\mu_{2}$ generates $H_{1}\left(M_{2}, \mathbb{Z}_{2}\right)$, we must have $h \circ \rho_{2}\left(\mu_{2}\right)=1$ and $\rho_{2}\left(\mu_{2}\right)$ has order two.

We claim that since $M_{2}$ is a knot complement in a $\mathbb{Z} / 2 \mathbb{Z}$-homology sphere, if $K$ is the kernel of the unique surjection $\pi_{1}\left(M_{2}\right) \rightarrow \mathbb{Z} / 2 \mathbb{Z}$, then $\lambda_{2} \in[K, K]$. Consider a Seifert surface $S$ for $M$. The surface $S$ has $[\partial S]$ equal to $\left[\lambda_{2}\right]$ in $H_{1}(\partial M)$. We can explicitly construct the cover $M_{2}^{\prime}$ corresponding to $K$ by gluing together two copies of $M_{2}$ cut along $S$. Thus we see that $S$ lifts to $M_{2}^{\prime}$. This shows that the boundary of $S$, namely $\left[\lambda_{2}\right]$, is 0 in $H_{1}\left(M_{2}^{\prime}, \mathbb{Z}\right)$. Thus $\lambda_{2}$ is in $[K, K]$. Therefore, as $\rho_{2}(K)$ is an abelian group of isometries fixing $\operatorname{fix}\left(G^{\prime}\right)$, we have $\rho_{2}\left(\lambda_{2}\right)=I$. So the subgroup of $H$ generated by the images of $\left(\mu_{2}, \lambda_{2}\right)$ is finite, in fact has order 2. Thus $H$ itself is finite.

Now we'll argue that $H$ is infinite, yielding a contradiction. Look at $M_{1}$ and in particular at $\rho_{1}\left(\pi_{1}\left(\partial M_{1}\right)\right)$. Let $\mu_{1}$ be a meridian in $\pi_{1}\left(\partial M_{1}\right)$. As $\rho_{1}$ is nonintegral, it is easy to see from the homomorphism $x_{\rho_{1}}: \pi_{1}\left(M_{1}\right) \rightarrow \mathbb{C}^{\times}$that the $\gamma$ in $\pi_{1}\left(M_{1}\right)$ with non-integral trace are exactly those $\gamma$ which are non-zero in $H_{1}\left(M_{1}, \mathbb{Z}\right) /($ torsion$)$. Therefore, $\rho\left(\mu_{1}\right)$ has non-integral trace. In particular, $\rho_{1}\left(\mu_{1}\right)$ has infinite order, and hence $\rho_{1}\left(\pi_{1}\left(\partial M_{1}\right)\right)$ is infinite. As $\pi_{1}\left(\partial M_{1}\right)$ shares a finite index subgroup with $\pi_{1}\left(\partial M_{2}\right), H$ shares a finite index subgroup with $\rho_{1}\left(\pi_{1}\left(\partial M_{1}\right)\right)$. Thus $H$ is infinite. But we've already shown that $H$ is finite. This is a contradiction, and so $\rho_{2}$ must be reducible. This proves Claim 6.2 and thus the theorem.

\section{2-BRIDGE KNOTS TO WHICH THE THEOREM APPLIES}

Theorem 6.1 applies to many 2-bridge knots in $S^{3}$. A 2-bridge knot is determined by a pair of relatively prime odd integers $(p, q)$ with $0<q<p$ (for background see [BZb, §12], [HT]). In this section, we describe computations which show:

7.1. Theorem. Let $K(p, q)$ be a 2-bridge knot such that $p<40$. Let $M$ be the exterior of $K(p, q)$. If $M$ does not fiber over the circle, then $M$ satisfies the hypotheses of Theorem [6.1, and so $M$ is not commensurable to a fibered knot complement in a $\mathbb{Z} / 2 \mathbb{Z}$-homology sphere. 
Let $K(p, q)$ be a 2-bridge knot, and $M$ be its exterior. We will follow [HLM], where Hilden, Lozano, and Montesinos, building on work of Burde and Riley, give a simple method for computing the $\mathrm{PSL}_{2} \mathbb{C}$-character variety $X(M)$.

The standard presentation of $\pi_{1}(M)$ has as generators two elements $a$ and $b$, each of which is a meridian at the top of one of the two bridges. As $a$ and $b$ are conjugate, we can take coordinates on $X(M)$ to be $x=\operatorname{tr}_{a^{2}}$ and $z=\operatorname{tr}_{a b}$ (the latter makes sense even in $\mathrm{PSL}_{2} \mathbb{C}$ because $a$ and $b$ are conjugate). Thus $X(M)$ is a plane curve. There is a polynomial with integer coefficients $f(x, z)$ such that $X(M)$ is the set of points in $\mathbb{C}^{2}$ satisfying $f(x, z)=0$. Section 5 of [HLM] gives a simple recursive procedure for computing this polynomial.

Let $M$ be the complement of a 2-bridge knot which does not fiber. Because 2-bridge knots are alternating, this is equivalent to $\Delta_{M}$ being non-monic (see e.g. [BZb, §13.C]). To decide if $M$ satisfies the hypotheses of Theorem 6.1, we first need to factor the polynomial $f(x, z)$ into irreducible factors over $\mathbb{C}$, and determine which component is $X_{0}(M)$. Let $f_{0}(x, z)$ be the polynomial defining $X_{0}(M)$. It is easy to check that a character in $X(M)$ comes from a reducible representation if and only if $x=z$. So $X_{0}(M)$ contains a non-integral reducible representation if and only if the polynomial $g(x)=f_{0}(x, x)$ has a non-integral root.

So the hard part of checking whether Theorem 6.1 applies is determining the factor $f_{0}$ of $f$. First, since $f(x, z)$ has rational coefficients, there is an algorithm for factoring it over $\mathbb{C}$. This is because one can a priori determine a finite extension $k$ of $\mathbb{Q}$ such that the irreducible factors of $f$ over $k$ are the same as those of $f$ over $\mathbb{C}$. Take a rational line $L$ in $\mathbb{C}^{2}$ which has simple intersections with the algebraic set $V=\{f=0\}$ and such that $L$ and $V$ don't intersect at infinity in $\mathbb{C} \mathrm{P}^{2}$. Then take $k$ to be $\mathbb{Q}$ adjoin the coordinates of $L \cap V$. The factoring of a multivariable polynomial with coefficients in a number field is a well-studied problem (for surveys see Kal1, Kal2 ). It is worth mentioning that $f$ sometimes factors into more components over $\mathbb{C}$ than over $\mathbb{Q}$, the lexicographically smallest example being $K(45,29)$. The computations for Theorem 7.1 were done using the computer algebra system Maple Wat], which has a built in procedure for factoring polynomials over $\mathbb{C}$.

For most of the 2-bridge knots included in Theorem [7.1, every factor $f_{i}$ of $f$ contained a non-integral reducible representation, and so it was not necessary to determine which $f_{i}$ defined $X_{0}(M)$. In the exceptional cases $\{(15,11),(27,5)$, $(27,11),(27,17),(27,19),(33,23),(35,29)\}$, we used the result of Section 6.4 of [HLM], who determined $f_{i}$ for $p<40$ ([HLM] gives an algorithm for determining $f_{0}$ in general, but it is quite involved).

Finally, to apply Theorem 6.1 we have to check that $M$ is generic. Reid [Rei, §4] showed that the only arithmetic knot complement in $S^{3}$ is the figure- 8 complement, which fibers. So $M$ is non-arithmetic. We also need to check that the cusp of the commensurator is non-rigid. We did this by checking that the cusp shape is not in $\mathbb{Q}(i)$ or $\mathbb{Q}(\sqrt{-3})$. Let $\Gamma \subset \mathrm{PSL}_{2} \mathbb{C}$ be the image of the discrete faithful representation of $\pi_{1}(M)$. Conjugate $\Gamma$ so that the meridian generators are:

$$
a=\left(\begin{array}{ll}
1 & 1 \\
0 & 1
\end{array}\right) \quad \text { and } \quad b=\left(\begin{array}{ll}
1 & 0 \\
u & 1
\end{array}\right)
$$

for some $u \in \mathbb{C}$. Riley showed that $u$ is always an algebraic integer [Ril §3]. Thus $\Gamma$ consists solely of matrices with algebraic integer entries. A longitudein the same 
copy of $\pi_{1}(\partial M)$ as $a$ has the form

$$
\left(\begin{array}{ll}
1 & \tau \\
0 & 1
\end{array}\right) .
$$

The cusp shape of $M$ is $\tau$, and so the cusp shape of $M$ is always an algebraic integer. The integers in $\mathbb{Q}(i)$ and $\mathbb{Q}(\sqrt{-3})$ are discrete, and so it's easy to check numerically using SnapPea $[\mathrm{W}]$ that the cusp shape of $M$ is not in $\mathbb{Q}(i)$ or $\mathbb{Q}(\sqrt{-3})$, and hence that $M$ is generic.

It would have been nicer to prove that every non-fibered 2-bridge knot satisfies the hypothesis of Theorem 6.1 but this seems a difficult thing to do-in some cases there are components of $X(M)$ which contain no reducible representations, and it is hard to see any special property $X_{0}(M)$ that would prevent this from happening there.

7.2. Remark. Actually, worrying about how $f$ splits up over $\mathbb{C}$ as opposed to $\mathbb{Q}$ is not really necessary. The character varieties and all the maps between them in the proof of Theorem 6.1 are all defined over $\mathbb{Q}$. Thus we could weaken the hypothesis of having a non-integral reducible representation in $X_{0}\left(M_{1}\right)$ to having such a representation in the $\mathbb{Q}$-irreducible component of $X(M)$ containing $X_{0}\left(M_{1}\right)$.

\section{EXAMPLES OF FIBERED AND NON-FIBERED PAIRS}

8.1. The dodecahedral knots. The two dodecahedral knots $D_{f}$ and $D_{s}$ were introduced by Aitchison and Rubinstein in $\left[\mathrm{AR}\right.$. They are a pair of knots in $S^{3}$. The complements $M_{f}$ and $M_{s}$ are hyperbolic and both are quotients of $\mathbb{H}^{3}$ by subgroups of the symmetry group of the tiling of $\mathbb{H}^{3}$ by $\{5,3,6\}$-ideal dodecahedra. Thus $M_{f}$ and $M_{s}$ are commensurable. In [AR], they show that $M_{f}$ is fibered. On the other hand, $M_{s}$ is not fibered because its Alexander polynomial is non-monic:

$$
\begin{aligned}
\Delta_{M_{s}}= & 25 t^{4}-250 t^{3}+1035 t^{2}-2300 t+2981 \\
& -2300 t^{-1}+1035 t^{-2}-240 t^{-3}+25 t^{-4} .
\end{aligned}
$$

The commensurator of $M_{f}$ and $M_{s}$ has a rigid cusp, and so $D_{f}$ and $D_{s}$ are not generic (though they are non-arithmetic). So the hypotheses of Theorem 6.1 are not satisfied by $M_{s}$.

8.2. Small volume examples. Here is an example of two 1-cusped manifolds which are commensurable where one is fibered and the other not. The two manifolds are $M_{1}=m 035$ and $M_{2}=m 039$ from the Callahan-Hildebrand-Weeks census CHW]. These manifolds have the same volume, 3.177293278..., and same first homology group, $\mathbb{Z} / 4 \mathbb{Z} \oplus \mathbb{Z}$. Weeks' computer program SnapPea $[\mathrm{W}]$, checks that $M_{1}$ and $M_{2}$ have a common 2 -fold cover. Presentations for the manifolds' fundamental groups are:

$$
\pi_{1}\left(M_{1}\right)=\left\langle a, b \mid a b^{3} a^{-2} b^{3} a b^{-2}\right\rangle \text { and } \pi_{1}\left(M_{2}\right)=\left\langle a, b \mid a b^{4} a b^{-1} a^{-1} b^{2} a^{-1} b^{-1}\right\rangle .
$$

An easy calculation shows that the Alexander polynomials are:

$$
\Delta_{M_{1}}=3 t-2+3 t^{-1} \text { and } \Delta_{M_{2}}=t-6+t^{-1} .
$$

Because of the lead coefficient of $\Delta_{M_{1}}$, the manifold $M_{1}$ does not fiber over $S^{1}$. On the other hand, $M_{2}$ is the punctured torus bundle over $S^{1}$ with monodromy $+L^{4} R$.

Goodman's program Snap G], calculates that these manifolds are not arithmetic and that the cusp field is a cubic extension of $\mathbb{Q}$. Thus they are generic. It is not 
too hard to check that there are non-integral reducible representations on $X_{0}\left(M_{1}\right)$. Thus this example shows that the hypothesis of Theorem 6.1 requiring knot complements in a $\mathbb{Z} / 2 \mathbb{Z}$-homology sphere is necessary. Some further examples among the census manifolds are:

- The pair $(m 037, m 040)$ have a common 2-fold cover and $m 037$ doesn't fiber but $m 040$ does. This pair is also commensurable with $m 035$ and $m 039$ via 4-fold covers.

- The pair $(m 139, m 140)$ have a common 4 -fold cover, and $m 139$ doesn't fiber but $m 140$ does. Both of these manifold are arithmetic and so not generic.

None of these examples are knot complements in $\mathbb{Z} / 2 \mathbb{Z}$-homology spheres.

The strategy for finding these examples was this. First, we used the data provided with Snap to get a list of census manifolds grouped by commensurability invariants. Then we used Lackenby's taut ideal triangulations [Lac to identify many census manifolds which fiber over $S^{1}$. From this, we selected pairs of manifolds whose trace field and cusp density were the same, one of which fibered and the other of which did not appear to fiber. Most of the census manifolds fiber, making examples rare.

8.3. Surgeries on the Whitehead link. Let $W$ be the complement of the Whitehead link in $S^{3}$. Let $W(a, b)$ denote the 1-cusped manifold obtained by filling in one of the two cusps of $W$ via $(a, b)$ Dehn filling. Hodgson, Meyerhoff, and Weeks gave a very elegant construction of a family of fibered/non-fibered pairs which are fillings of $W[\mathrm{HMW}]$. They showed that

8.4. Theorem. Let $m \in \mathbb{Z}$ be a multiple of $4, m \notin\{0,4\}$. Then

$$
M_{1}=W(m,-1-(m / 2)) \quad \text { and } \quad M_{2}=W(m,-1)
$$

are a pair of 1-cusped hyperbolic 3-manifolds such that:

- $M_{1}$ and $M_{2}$ have a common 2-fold cover with two cusps.

- $M_{1}$ does not fiber over $S^{1}$ because its Alexander polynomial is not monic.

- $M_{2}$ fibers over $S^{1}$, as it is the punctured torus bundle with monodromy $\pm R L^{m}$.

These examples overlap with the ones in the preceding section. Namely, the pairs $(m 035, m 039)$ and $(m 037, m 040)$ are of this type. The manifold $M_{1}$ doesn't satisfy the hypotheses of Theorem 6.1 because $H_{1}\left(M_{1} ; \mathbb{Z}\right)=\mathbb{Z} \oplus \mathbb{Z} / m \mathbb{Z}$. As $m$ is divisible by $4, M_{1}$ is not a knot complement in a $\mathbb{Z} / 2 \mathbb{Z}$-homology sphere.

\section{REFERENCES}

[AR] I. R. Aitchison and J. H. Rubinstein, Combinatorial cubings, cusps, and the dodecahedral knots, Topology '90 (Columbus, OH, 1990), de Gruyter, Berlin, 1992, pp. 17-26. MR 93i:57016

[BNS] Robert Bieri, Walter D. Neumann, and Ralph Strebel, A geometric invariant of discrete groups, Invent. Math. 90 (1987), no. 3, 451-477. MR 89b:20108

[Bor] A. Borel, Commensurability classes and volumes of hyperbolic 3-manifolds, Ann. Scuola Norm. Sup. Pisa Cl. Sci. (4) 8 (1981), no. 1, 1-33. MR 82j:22008

[Bro] Kenneth S. Brown, Trees, valuations, and the Bieri-Neumann-Strebel invariant, Invent. Math. 90 (1987), no. 3, 479-504. MR 89e:20060

[BW] Michel Boileau and Shicheng Wang, Non-zero degree maps and surface bundles over $S^{1}$, J. Differential Geom. 43 (1996), no. 4, 789-806. MR 98g:57023

[BZa] S. Boyer and X. Zhang, On Culler-Shalen seminorms and Dehn filling, Ann. of Math. (2) 148 (1998), no. 3, 737-801. MR 2000d:57028 
[BZb] Gerhard Burde and Heiner Zieschang, Knots, Walter de Gruyter \& Co., Berlin, 1985. MR 87b:57004

[CGLS] Marc Culler, C. McA. Gordon, J. Luecke, and Peter B. Shalen, Dehn surgery on knots, Ann. of Math. (2) 125 (1987), no. 2, 237-300. MR 88a:57026

[CHW] Patrick J. Callahan, Martin V. Hildebrand, and Jeffrey R. Weeks, A census of cusped hyperbolic 3-manifolds, Math. Comp. 68 (1999), no. 225, 321-332. MR 99c:57035]

[CS] Marc Culler and Peter B. Shalen, Varieties of group representations and splittings of 3-manifolds, Ann. of Math. (2) 117 (1983), no. 1, 109-146. MR 84k:57005

[Dun] Nathan M. Dunfield, Alexander and Thurston norms of 3-manifolds fibering over the circle, Pacific J. Math 200 (2001), no. 1, 43-58, arXiv:math.GT/9908050.

[G] O. Goodman, Snap, http://www.ms.unimelb.edu.au/ snap/.

[HLM] Hugh M. Hilden, María Teresa Lozano, and José María Montesinos-Amilibia, On the arithmetic 2-bridge knots and link orbifolds and a new knot invariant, J. Knot Theory Ramifications 4 (1995), no. 1, 81-114. MR 96a:57019

[HMW] Craig D. Hodgson, G. Robert Meyerhoff, and Jeffrey R. Weeks, Surgeries on the Whitehead link yield geometrically similar manifolds, Topology '90 (Columbus, OH, 1990), de Gruyter, Berlin, 1992, pp. 195-206. MR 93i:57019

[HT] A. Hatcher and W. Thurston, Incompressible surfaces in 2-bridge knot complements, Invent. Math. 79 (1985), no. 2, 225-246. MR 86g:57003

[Kal1] E. Kaltofen, Polynomial factorization, Computer Algebra (B. Buchberger, G. Collins, and R. Loos, eds.), Springer Verlag, Heidelberg, 2 ed., 1982, pp. 95-113.

[Kal2] Erich Kaltofen, Polynomial factorization 1982-1986, Computers in mathematics (Stanford, CA, 1986), Dekker, New York, 1990, pp. 285-309. MR 92f:12001

[Kir] Rob Kirby, Problems in low-dimensional topology, Geometric topology (Athens, GA, 1993), Amer. Math. Soc., Providence, RI, 1997, http://www.math.berkeley.

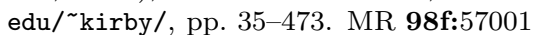

[Lac] Marc Lackenby, Taut ideal triangulations of 3-manifolds, Geom. Topol. 4 (2000), 369395, arXiv:math.GT/0003132. MR 1790190

[LR] D. D. Long and A. W. Reid, Commensurability and the character variety, Math. Res. Lett. 6 (1999), no. 5-6, 581-591. MR 2000m:57017

[Lub] Alexander Lubotzky, Subgroup growth and congruence subgroups, Invent. Math. 119 (1995), no. 2, 267-295. MR 95m:20054

[McM] Curtis T. McMullen, The Alexander polynomial of a 3-manifold and the Thurston norm on cohomology, to appear in Ann. Sci. École. Norm. Sup. (4).

[Rei] Alan W. Reid, Arithmeticity of knot complements, J. London Math. Soc. (2) 43 (1991), no. 1, 171-184. MR 92a:57011

[Ril] Robert Riley, Parabolic representations of knot groups. I, Proc. London Math. Soc. (3) 24 (1972), 217-242. MR 45 \#9313

[Rol] Dale Rolfsen, Knots and links, Publish or Perish Inc., Berkeley, Calif., 1976, Mathematics Lecture Series, No. 7. MR 58 \#24236

[Sha] P. B. Shalen, Representations of 3-manifold groups, Handbook of geometric topology, Elsevier Press, to appear.

[Sta] John Stallings, On fibering certain 3-manifolds, Topology of 3-manifolds and related topics (Proc. The Univ. of Georgia Institute, 1961), Prentice-Hall, Englewood Cliffs, N.J., 1962, pp. 95-100. MR 28 \#1600

[Thu] William P. Thurston, Three-dimensional manifolds, Kleinian groups and hyperbolic geometry, Bull. Amer. Math. Soc. (N.S.) 6 (1982), no. 3, 357-381. MR 83h:57019

[Tur] V. G. Turaev, The Alexander polynomial of a three-dimensional manifold, Math. USSR Sbornik 26 (1975), 313-329. MR 52 \#4306

[W] J. Weeks, SnapPea, http://www.northnet.org/weeks/.

[Wat] Waterloo Maple Software, Maple 6, 2000.

Department of Mathematics, Harvard University, Cambridge Massachusetts 02138 E-mail address: dannyc@math.harvard.edu

Department of Mathematics, Harvard University, Cambridge Massachusetts 02138

E-mail address: nathand@math.harvard.edu 